\title{
J. Pellaumail
}

\section{Disponibilité d'un ensemble complexe}

Revue française d'automatique, d'informatique et de recherche opérationnelle. Recherche opérationnelle, tome 14, n 2 (1980), p. 137-145.

<http://www.numdam.org/item?id=RO_1980_14_2_137_0>

(C) AFCET, 1980, tous droits réservés.

L'accès aux archives de la revue « Revue française d'automatique, d'informatique et de recherche opérationnelle. Recherche opérationnelle » implique l'accord avec les conditions générales d'utilisation (http://www.numdam.org/ legal.php). Toute utilisation commerciale ou impression systématique est constitutive d'une infraction pénale. Toute copie ou impression de ce fichier doit contenir la présente mention de copyright.

\section{NumDam}

Article numérisé dans le cadre du programme

Numérisation de documents anciens mathématiques

http://www.numdam.org/ 


\title{
DISPONIBILITÉ D'UN ENSEMBLE COMPLEXE (*)
}

\author{
par J. Pellaumail $\left({ }^{1}\right)$
}

\begin{abstract}
Résumé. - On établit une formule simple explicite donnant l'indisponibilité transitoire d'un ensemble série-parallèle d'équipements. Chaque équipement comporte des cartes échangeables en stocks limités. La formule donnée est particulièrement bien adaptée aux calculs d'optimisation. L'approximation effectuée correspond à une erreur relative sur l'indisponibilité de l'ordre de l'indisponibilité elle-même.
\end{abstract}

Abstract. - The availability of a series-parallel system is studied. Some explicit formulas are stated. These formulas are very convenient to get the optimal inventory values.

\section{SYSTÈME GLOBAL}

Le système global considéré est constitué de $r$ ensembles fonctionnant en série en ce sens que le système global est considéré comme défaillant dès que l'un des ensembles considérés est défaillant.

Par exemple il peut s'agir des diverses parties constitutives d'un central téléphonique : multi-enregistreurs, taxeurs, marqueurs, traducteurs, etc. Le central est défaillant si l'un de ces sous-ensembles est défaillant.

Soit, alors, pour $1 \leqq i \leqq r$, la probabilité $g(i, t)$, à l'instant $t$, que l'ensemble $i$ soit défaillant. Si $g(t)$ est la probabilité que, à l'instant $t$, le système global soit défaillant, on a

$$
1-g(t)=\prod_{i=1}^{r}[1-g(i, t)] .
$$

En première approximation, on peut prendre

$$
g(t) \simeq \sum_{i=1}^{r} g(i, t) .
$$

(*) Reçu décembre 1978.

(1) I.N.S.A., Rennes (France).

R.A.I.R.O. Recherche opérationnelle/Operations Research, 0399-0842/1980/137/\$5.00

(C) AFCET-Bordas-Dunod 
L'indisponibilité moyenne vaut alors :

$$
\bar{g}(t)=\frac{1}{T} \int_{0}^{T} g(t) d t \simeq \sum_{i=1}^{r} \frac{1}{T} \int_{0}^{T} g(i, t) d t,
$$

c'est-à-dire la somme des indisponibilités moyennes de chaque ensemble.

On est donc ramené au calcul de l'indisponibilité moyenne de chaque ensemble.

Rappelons aussi que l'approximation $g(t) \simeq \sum_{i=1}^{r} g(i, t)$ est satisfaite avec une erreur relative inférieure à $1 / 2 \sum_{i=1}^{r} g(i, t)$; de plus, on a toujours $g(t) \leqq \sum_{i=1}^{r} g(i, t)$.

\section{2. ÉTUDE D'UN ENSEMBLE}

Le problème est d'étudier la disponibilité d'un ensemble constitué de $n$ machines identiques fonctionnant en parallèle et toutes constamment sous tension; on considère que cet ensemble est en panne dès que $m$ machines sont en panne ( $m$ est donc une donnée technologique); dans la suite on supposera $m \leqq 3$, mais la méthodologie proposée reste valable pour $m>3$.

Chaque machine comporte $q$ modules, tous distincts : notons que la méthodologie proposée resterait utilisable si les modules ne sont pas tous distincts.

Pour le module de type $i$, il existe un stock de $s_{i}$ modules de rechange, en plus des modules dans les $n$ machines (on peut avoir $s_{i}=0$ ).

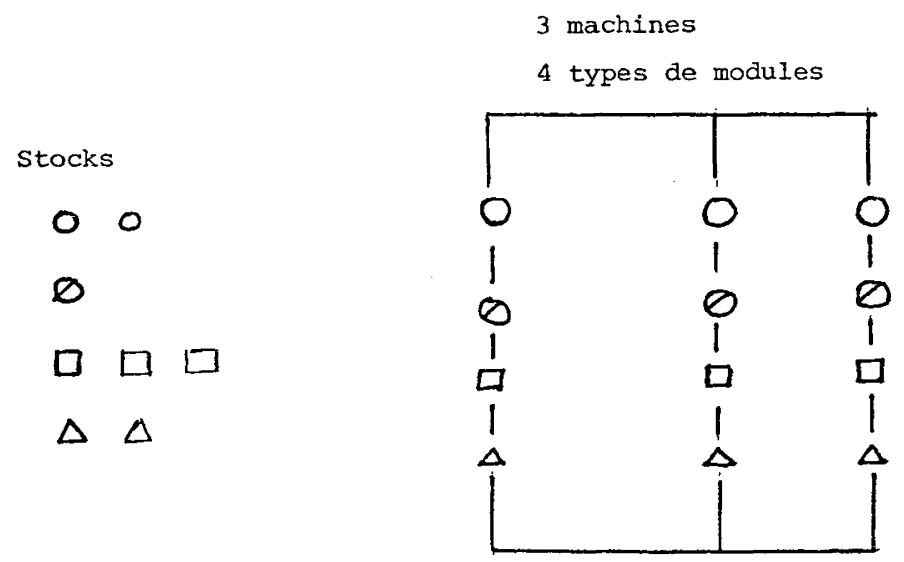

R.A.I.R.O. Recherche opérationnelle/Operations Research 
La figure correspond au cas où l'ensemble comprend trois machines identiques qui fonctionnent en parallèles et qui sont constituées chacune de quatre types de modules, ici symbolisés respectivement par des ronds, des ronds barrés, des carrés et des triangles. Par exemple, cet ensemble peut correspondre aux multi-enregistreurs du central téléphonique : l'ensemble est considéré comme défaillant si deux multi-enregistreurs sont en panne; les modules sont les diverses cartes du multi-enregistreur, le câblage étant considéré comme l'un de ces modules.

Chaque module tombe en panne suivant la loi exponentielle de taux $x_{i}$ (ce taux dépend donc du type $i$ du module considéré). Quand un module tombe en panne, celui-ci est échangé jusqu'à épuisement du stock. La durée de l'indisponibilité due à un tel échange (quand le stock le permet) suit la loi exponentielle de taux $y_{i}$.

Le cannibalisme n'est pas autorisé en ce sens qu'il n'est pas possible d'aller chercher dans une machine, par ailleurs en panne, un module en bon état pour le mettre sur une autre machine.

Pour chaque machine, les modules sont en série en ce sens que la machine est en panne dès que l'un des modules est en panne.

L'ensemble est mis en marche à l'instant $t=0$.

Soit $g_{m}(t)$ la probabilité pour que l'ensemble soit en panne à l'instant $t \geqq 0$ ( $m$ étant définie comme indiqué précédemment). On se propose de calculer l'indisponibilité moyenne sur l'intervalle de temps $[0, T]$, c'est-à-dire la quantité

$$
\bar{g}_{m}(T)=\frac{1}{T} \int_{0}^{T} g_{m}(t) d t
$$

Ce qui nous semble intéressant dans l'étude qui suit, c'est de noter que l'on peut donner la valeur explicite de $\bar{g}_{m}(T)$ dans le cas (le plus fréquent dans la pratique) où les taux $x_{i}$ sont faibles; notamment, on supposera que l'on peut confondre

$$
1-\exp .\left\{-\sum_{i=1}^{q} x_{i} T\right\} \quad \text { et } \quad \sum_{i=1}^{q} x_{i} T \text {. }
$$

Par contre on supposera $y_{i} T$ grand $(>10)$ pour tout élément $i$ de $I$.

\section{3. ÉTUDE DES STOCKS POUR UN MODULE}

On suppose qu'il y a $n$ machines en redondance utilisant un module de taux de mortalité $x$; le niveau du stock vaut $s$ (en plus des $n$ modules présents dans les machines) à l'instant $t=0$. 
Dans toute la suite, on pose $a \wedge b=\min .\{a, b\}$.

On se propose ici de rappeler comment on calcule :

$p_{t}(k)=$ proba qu'à l'instant $t$ il n'y ait plus que $(n+s-k)$ modules en bon état (y compris les $n$ modules en place sur les machines), autrement dit $k$ modules sont déjà tombés en panne.

On a $p_{0}(0)=1$ et (équations de Chapman-Kolmogoroff) :

$$
p_{t}^{\prime}(k)=-p_{t}(k) \cdot x \cdot[(n+s-k) \wedge n]+p_{t}(k-1) \cdot x \cdot[(n+s-k+1) \wedge n] .
$$

On en déduit, pour $k \leqq s$ :

$$
p_{t}(k)=\frac{(n x t)^{k}}{k !} e^{-(n x t)} \quad \text { (formule classique de Poisson). }
$$

Compte tenu des valeurs faibles de nxt par rapport à 1 , on peut prendre, avec une très bonne approximation.

$$
p_{t}(k)=\frac{(n x t)^{k}}{k !} \text {. }
$$

Pour $k>s$, la formule exacte est nettement plus compliquée, mais en utilisant l'approximation ci-dessus, on obtient facilement, dans tous les cas (avec $k \geqq 1$ ):

$$
\sum_{j \geqq k} p_{t}(j)=\frac{(x t)^{k}}{k !} \prod_{j=1}^{k}[n \wedge(n+s-j+1)] .
$$

Dans la suite, on posera (pour $k \geqq 1$ ) :

$$
a(n, s, k)=\prod_{j=1}^{k}[n \wedge(n+s-j+1)] \frac{1}{j},
$$

ce qui donne

$$
\sum_{j \geqq k} p_{t}(j)=(x t)^{k} a(n, s, k) .
$$

On constate facilement que, pour $k>1+s$, la «formule de Poisson " n'est absolument pas satisfaisante.

\section{CAS $m=1$ (pas de redondance)}

On désigne par $b_{i}$ la probabilité pour que le module de type $i$ soit en cours d'échange (sachant que le stock n'est pas épuisé); il est facile de vérifier que

$$
b_{i} \leqq \frac{x_{i}}{x_{i}+y_{i}} .
$$

R.A.I.R.O. Recherche opérationnelle/Operations Research 
On pose

$$
b=\sum_{i=1}^{q} b_{i} .
$$

On désigne par $d_{1}(i, t)$ la probabilitc pour que, à l'instant $t$, le module de type $i$ soit en rupture de stock avec « une demande différée », c'est-à-dire que $\left(1+s_{i}\right)$ modules de type $i$ sont tombés en panne.

Compte tenu de ce qui précède, on a

$$
d_{1}(i, t)=\left(x_{i} t\right)^{1+s_{i}} a\left(n, s_{i}, 1+s_{i}\right) .
$$

On pose

$$
d_{1}(t)=\sum_{i=1}^{q} d_{1}(i, t)
$$

Si on suppose $m=1$, on a

$$
g_{1}(t) \simeq n b+d_{1}(t)
$$

En fait $g_{1}(t)$ est un peu plus petit que cela mais, si les taux $x_{i}$ sont faibles, l'approximation ci-dessus est excellente.

On pose

$$
u_{i}=\frac{1}{\left(2+s_{i}\right) !}\left(x_{i} T\right) n^{1+s_{i}}
$$

ce qui donne

$$
\vec{g}_{i}(T)=n b+\sum_{i=1}^{q} u_{i} .
$$

5. CAS $m=2$ (on tolère une panne et une seule).

On désigne par $d_{2}(i, t)$ la probabilité pour que, à l'instant $t$, le module de type $i$ soit en rupture de stock avec « deux demandes différées », c'est-à-dire que $\left(2+s_{i}\right)$ modules de type $i$ sont tombés en panne. On a donc

$$
d_{2}(i, t)=\left(x_{i} t\right)^{2+s_{i}} a\left(n, s_{i}, 2+s_{i}\right) .
$$

On pose

$$
d_{2}(t)=\sum_{i=1}^{q} d_{2}(i, t)
$$

vol. $14, \mathrm{n}^{\circ} 2$, mai 1980 
Si on suppose $m=2$, on a

$$
g_{2}(t) \simeq d_{2}(t)+\frac{1}{2}\left[d_{1}(t)\right]^{2}+d_{1}(t)(n-1) b+C_{n}^{2} b^{2},
$$

en notant $C_{n}^{2}$ le coefficient du binôme habituel; en fait, $g(t)$ est un peu plus petit car certaines défaillances sont «comptées deux fois », mais l'approximation ainsi effectuée est excellente si, pour tout $i, x_{i}$ est assez petit devant $\sum_{i=1}^{q} x_{i}$; de plus, dans tous les cas, $g_{2}(t)$ est plus petit que la valeur indiquée, c'est-à-dire qu'on a une majoration de l'indisponibilité.

Comme précédemment, on utilise le fait que la probabilité d'une réunion d'événements est plus petite que la somme des probabilités de ces divers événements et le fait que la probabilité de l'intersection de deux événements indépendants est égale au produit des probabilités de chacun de ces événements.

Le premier terme $d_{2}(t)$ correspond au cas où les deux machines sont en panne par défaut d'un même module.

Le deuxième terme $1 / 2\left[d_{1}(t)\right]^{2}$ correspond au cas où deux machines sont en panne par rupture de stock pour deux modules différents. On peut diviser par deux, sinon les diverses possibilités de double panne sont comptées deux fois.

Le troisième terme $d_{1}(t)(n-1) b$ correspond au cas où une machine est en panne par rupture de stock et une autre parce qu'il faut changer un module.

Le quatrième terme correspond au cas où les deux machines sont en panne parce qu'il faut, sur chacune, procéder à un changement de module. On a donc

$$
\begin{aligned}
& g_{2}(t) \simeq \sum_{i=1}^{q}\left(x_{i} t\right)^{2+s_{i}} a\left(n, s_{i}, 2+s_{i}\right) \\
& +\frac{1}{2} \sum_{i=1}^{q} \sum_{j=1}^{q}\left(x_{i} t\right)^{1+s_{i}}\left(x_{j} t\right)^{1+s_{j}} a\left(n, s_{i}, 1+s_{i}\right) a\left(n, s_{j}, 1+s_{j}\right) \\
& \quad+(n-1) b \sum_{i=1}^{q}\left(x_{i} t\right)^{1+s_{i}} a\left(n, s_{i}, 1+s_{i}\right)+\frac{1}{2} n(n-1) b^{2} .
\end{aligned}
$$

En fait, dans le deuxième terme, on peut se limiter aux termes de la somme double tels que $i \neq j$ ce qui est encore un peu plus précis.

On pose

$$
\begin{gathered}
a_{i}^{\prime}:=\left(x_{i} T\right)^{1+s_{i}} a\left(n, s_{i}, 1+s_{i}\right)=\left(x_{i} T\right)^{1+s_{i}} \frac{1}{\left(1+s_{i}\right) !} n^{1+s_{i}}, \\
a_{i}^{\prime \prime}:=\left(x_{i} T\right)^{2+s_{i}} \frac{1}{\left(2+s_{i}\right) !}(n-1) n^{1+s_{i}}, \\
v_{i}:=\frac{1}{3+s_{i}} a_{i}^{\prime \prime}+(n-1) b \frac{1}{2+s_{i}} a_{i}^{\prime}
\end{gathered}
$$

R.A.I.R.O. Recherche opérationnelle/Operations Research 
On a alors :

$$
v_{i}^{\prime}:=a_{i}^{\prime} \cdot \sum_{j \neq i} \frac{1}{3+s_{i}+s_{j}} a_{j}^{\prime} .
$$

$$
\bar{g}_{2}(T) \simeq \frac{1}{2} n(n-1) b^{2}+\sum_{i=1}^{q}\left(v_{i}+\frac{1}{2} v_{i}^{\prime}\right)
$$

6. CAS $m=3$ (on tolère deux pannes et deux seulement)

On désigne par $d_{3}(i, t)$ la probabilité pour que, à l'instant $t$, le module de type $i$ soit en rupture de stock avec « trois demandes différées », soit :

$$
d_{3}(i, t)=\left(x_{i} t\right)^{3+s_{i}} a\left(n, s_{i}, 3+s_{i}\right)
$$

On pose

$$
d_{3}(t)=\sum_{i=1}^{q} d_{3}(i, t)
$$

Si on suppose $m=3$, en procédant comme précédemment, on a

$$
\begin{aligned}
g_{3}(t) \simeq d_{3}(t)+d_{1}(t) & . d_{2}(t)+\frac{1}{3 !}\left[d_{1}(t)\right]^{3} \\
& +d_{2}(t)(n-1) b+\frac{1}{2}\left[d_{1}(t)\right]^{2}(n-2) b+d_{1}(t) C_{n}^{2} \cdot b^{2}+C_{n}^{3} b^{3} .
\end{aligned}
$$

Le deuxième terme, par exemple, correspond au cas où une machine est en panne par « simple » rupture de stock et une autre par " double » rupture de stock.

De même, le quatrième terme $d_{2}(t)(n-1) b$ correspond au cas où deux machines sont en panne par "double " rupture des stocks pour un même module et la troisième machine est en panne parce qu'on échange un module.

En fait, comme précédemment, on peut être encore un peu plus précis en excluant certains doubles produits.

En prenant $a_{i}^{\prime \prime \prime}=\left(x_{i} T\right)^{3+s_{i}} a\left(n, s_{i}, 3+s_{i}\right)$ et en raisonnant comme précédemment, on a

$$
\begin{aligned}
& \bar{g}_{3}(T) \simeq \sum_{i=1}^{q} \frac{1}{4+s_{i}} a_{i}^{\prime \prime \prime}+\sum_{i=1}^{q} \sum_{j=1}^{i-1} \frac{1}{4+s_{i}+s_{j}} a_{i}^{\prime} a_{j}^{\prime \prime} \\
&+\sum_{i=1}^{q} \sum_{j=1}^{i-1} \sum_{k=1}^{j-1} \frac{1}{4+s_{i}+s_{j}+s_{k}} a_{i}^{\prime} a_{j}^{\prime} a_{k}^{\prime} \\
&+(n-1) b \sum_{i=1}^{q} \frac{1}{3+s_{i}} a_{i}^{\prime \prime}+(n-1) b \sum_{i=1}^{q} \sum_{j=1}^{i-1} \frac{1}{3+s_{i}+s_{j}} a_{i}^{\prime} a_{j}^{\prime} \\
&+\frac{1}{2} n(n-1) b^{2} \sum_{i=1}^{q} \frac{1}{2+s_{i}} a_{i}^{\prime}+\frac{1}{6} n(n-1)(n-2) b^{3} .
\end{aligned}
$$

vol. $14, \mathrm{n}^{\circ} 2$, mai 1980 
Insistons bien sur le fait que, dans tous les cas, $\bar{g}_{3}(T)$ est majoré par l'expression donnée ci-dessus et que cette expression constitue une excellente approximation de $\bar{g}_{3}(T)$ si les taux de défaillance sont assez faibles.

On peut d'ailleurs augmenter la précision de l'approximation effectuée en supprimant certains doubles produits ce qui conduit finalement à :

On définit $a_{i}^{\prime}$ et $a_{i}^{\prime \prime}$ comme plus haut et on pose

$$
\begin{gathered}
a_{i}^{\prime \prime \prime}:=\left(x_{i} T\right)^{3+s_{i}} \frac{1}{\left(3+s_{i}\right) !}(n-2)(n-1) n^{1+s_{i},} \\
w_{i}:=\frac{1}{4+s_{i}} a_{i}^{\prime \prime \prime}+(n-1) b \frac{1}{3+s_{i}} a_{i}^{\prime \prime}+\frac{1}{2} n(n-1) b^{2} \frac{1}{2+s_{i}} a_{i}^{\prime}, \\
w_{i}^{\prime}:=a_{i}^{\prime} \sum_{j \neq i, j=1}^{q}\left[\frac{1}{4+s_{i}+s_{j}} a_{j}^{\prime \prime}+(n-1) b \frac{1}{3+s_{i}+s_{j}} a_{j}^{\prime}\right]+a_{i}^{\prime \prime} \sum_{j \neq i, j=1}^{q} \frac{1}{4+s_{i}+s_{j}} a_{j}^{\prime}, \\
w_{i}^{\prime \prime}:=a_{i}^{\prime} \sum_{j \neq i, j=1}^{q} \sum_{l \neq i, l=1}^{j-1} \frac{1}{4+s_{i}+s_{j}+s_{l}} a_{j}^{\prime} a_{l}^{\prime} .
\end{gathered}
$$

On a alors :

$$
\vec{g}_{3}(T) \simeq \frac{1}{6} n(n-1)(n-2) b^{3}+\sum_{i=1}^{q}\left(w_{i}+\frac{1}{2} w_{i}^{\prime}+\frac{1}{3} w_{i}^{\prime \prime}\right) .
$$

\section{OPTIMISATION}

Les formules explicites simples données précédemment permettent notamment d'optimiser la politique des stocks avec des calculs relativement peu coûteux.

Considérons, par exemple, le cas $m=2$.

Augmenter d'une unité le niveau initial $s_{i}$ du stock associé au module de type $i$ revient quasiment à diminuer l'indisponibilité de la part qui était due (en tout ou partie) à ce module de type $i$, c'est-à-dire $\left(v_{i}+v_{i}^{\prime}\right)$. Les calculs qui précèdent nous ont donc donné, avec une très bonne approximation, la variation $z_{i}=\left(v_{i}+v_{i}^{\prime}\right)$ de l'indisponibilité quand on augmente $s_{i}$ d'une unité. De même, par exemple, si $m=3, z_{i}=w_{i}+w_{i}^{\prime}+w_{i}^{\prime \prime}$. On peut alors procéder à une optimisation suivant les techniques habituelles.

En général, il s'agit de minimiser l'indisponibilité sous une contrainte de coût donné.

R.A.I.R.O. Recherche opérationnelle/Operations Research 
Il faut noter qu'on a ici une situation particulière : pour un module donné, augmenter le stock d'une unité provoque une augmentation relative du coût associé à ce module inférieure à 2 et une diminution très forte de l'indisponibilité associée à ce module.

On peut donc conduire l'optimisation comme suit : on procède à une initialisation raisonnable; pour les modules dont le coût représente plus du centième du coût total, on considère les divers cas possibles raisonnables; pour les autres modules, on prend le plus petit niveau de stock $s_{i}$ tel que le rapport $c_{i} / z_{i}$ soit plus grand que $\lambda$; on initialise $\lambda$ par

$$
\lambda=\frac{\text { coût global initial }}{\text { indisponibilité globale initiale }}
$$

et on fait varier $\lambda$ jusqu'à ce que le coût global (après optimisation) soit le coût imposé. Il faut noter que $z_{i}$ dépend des valeurs $s_{j}$ pour $1 \leqq j \leqq q$; il faut donc, à chaque pas, réajuster le niveau $s_{i}$ du stock pour le module considéré. Avant de modifier $\lambda$, notamment au démarrage de l'algorithme, il faut donc s'accorder plusieurs itérations pour être sûr qu'on est arrivé aux " bonnes » valeurs des niveaux $s_{i}$.

Pour aider le décideur, il est souvent opportun de donner en plus de la solution " optimale » ainsi calculée, quelques autres solutions " voisines » obtenues en prenant des valeurs de $\lambda$ inférieures et supérieures à celles de la solution optimale.

\section{BIBLIOGRAPHIE}

D. R. Cox et H. D. Miller, The Theory of Stochastic Processes, Methuean and Co, 1968.

A. Khintchine, Desserte d'un grand nombre d'usagers, Dunod, Paris, 1969.

T. L. SAATY, Elements of Queueing Theory, McGraw-Hill Company, 1961. 